\title{
Detecting 17 Fine-Grained Dental Anomalies from Panoramic Dental Radiography using Artificial Intelligence
}

Sangyeon Lee ( $\sim$ sangyeonlee230@gmail.com )

Korea Advanced Institute of Science and Technology

Donghyun Kim

InvisionLab

Hogul Jeong

InvisionLab

\section{Research Article}

Keywords: Panoramic dental radiography, maxilla and mandibular area, abnormal signs, anonymized panoramic dental image.

Posted Date: October 11th, 2021

DOl: https://doi.org/10.21203/rs.3.rs-948048/v1

License: (a) (1) This work is licensed under a Creative Commons Attribution 4.0 International License. Read Full License

Version of Record: A version of this preprint was published at Scientific Reports on March 25th, 2022. See the published version at https://doi.org/10.1038/s41598-022-09083-2. 


\title{
Detecting 17 fine-grained dental anomalies from panoramic dental radiography using artificial intelligence
}

\author{
Sangyeon Lee ${ }^{1, *}$, Donghyun Kim ${ }^{2,3}$, Hogul Jeong² \\ ${ }^{1}$ Korea Advanced Institute of Science and Technology, Department of Bio and Brain Engineering, 34141, Daejeon, \\ Republic of Korea \\ ${ }^{2}$ InvisionLab, 05854, Seoul, Republic of Korea \\ ${ }^{3}$ Yeonsei University, Department of Advanced General Dentistry, 03722, Seoul, Republic of Korea \\ *Correspnding. sangyeonlee230@gmail.com
}

\begin{abstract}
Panoramic dental radiography is one of the most common examinations performed in dental clinics. Compared with other dental images, it covers a wide area from individual teeth to the maxilla and mandibular area. Dental clinicians can get much information about patientsôhealth. However, it is timeconsuming and laborious to detect all signs of anomalies because these regions are very complicated. So it is needed to filter out healthy images to save cliniciansôtime to examine. For this, we applied modern artificial intelligence-based computer vision techniques. In this study, we built a model to detect 17 fine-grained dental anomalies which are critical to patientsôdental health and quality of life. We used about 23,000 anonymized panoramic dental images taken from local dental clinics from July 2020 to July 2021. Our model can detect these abnormal signs and filter out normal images with high sensitivity of about 0.99 . The result indicates that our model can be used in real clinical practice to alleviate the burden of clinicians.
\end{abstract}

\section{Introduction}

Artificial intelligence techniques are fast developing and applied to various industries. Healthcare service is one of the most potential fields to take advantage of those techniques because of the fast accumulation of massive and complex features of medical data. Especially with the development of deep learning techniques, the convolutional neural network $(\mathrm{CNN})$ model and its variances are used in many fields of image analysis, such as classification and object detection. Because many medical data are collected in image formats, such as skin image datasets [1] and ophthalmoscope [2] images, artificial intelligence techniques are widely applied to various medical fields and tasks. Especially, regional convolutional neural network (RCNN) models are widely applied to the medical field in order to detect some medical signs from a given image to detect the region of breast cancer [3], pneumonia [4], and so on.

Dentistry is also one of the hospital branches where artificial intelligence techniques are vividly applied because it takes and uses a lot of medical images during a clinical routine. Patients admitted to hospitals are usually examined by radiographs. From simple intra-oral periapical x-rays to computed tomography, there are various tools to examine patients' oral health and status. Panoramic dental radiography is one of the most commonly performed imaging techniques by dentists and oral surgeons in everyday practice. It shows good patient acceptance because it is simple and painless and also uses a small amount of radial dose. [5] Panoramic dental radiography produces an image that includes not only maxillary and mandibular dental arches, which are mainly examined by dentists, but also the surrounding structures as the maxillary sinus, nasal fossa, temporomandibular joints, styloid processes, and hyoid bone. [6] Radiographic findings from these structures are suggested for diagnostic features of many diseases from dental anomalies to systemic diseases like hypoparathyroidism, hyperparathyroidism, and osteoporosis. [7] However, orthodontic and surrounding areas shown in panoramic dental radiography are complicated regions so correct diagnoses of anomalies can be very laborious and timeconsuming, also potentially inaccurate. [8] Also, dentists might only concentrate on teeth of symptoms and regions of interest due to lack of time. 
One of the methods to reduce this burden is computer-aided anomaly detection techniques. Diagnosis of dental anomalies with computational analysis of panoramic dental radiography is not a novel concept. Before the recent development of deep learning techniques, scientists tried to diagnose dental anomalies through image texture calculations $[9,10]$ or abnormality thresholding [11]. And with the recent advances of deep learning techniques, it has been quickly applied to increase the performance of analyzing panoramic dental radiography. Many of the studies are focused on detecting some signs of dental diseases from panoramic dental radiography. Especially, a large proportion of previous studies mainly selected carious lesions as their detection targets [9, 10, 11, 12, 13, 14], and few studies are focused on another dental anomaly, such as periodontal bone loss [15], odontogenic cyst, tumor [16], osteoporosis [17], impacted tooth [8], and so on. These studies used variations of convolutional neural network models developed for image classification or object detection tasks, such as MobileNet V2 [12], singlecolumn deep convolutional neural network (SC-DCNN) [17], regional convolutional neural network (RCNN) [13].

These previous studies could detect targeted disease with satisfying performance, however, most of the works targeted only one or a small number of diseases or abnormal signs. This limitation makes it hard to take one of the major advantages of panoramic dental radiography, which covers a wide area from individual teeth to mandibular and maxillary regions. Also, to reduce the burden of dental clinicians, it is needed to filter out healthy images to reduce the number of images that need to be manually examined.

Here, we selected fine-grained 17 anomalies that are closely related to patients' quality of life and also can be detected from panoramic dental radiography. We categorized them into four groups: carious lesions, calcifications, anomalies in dental regions, and anomalies in surrounding regions. To distinguish the dental region and surrounding regions, we used the method suggested by Langland, et al [18]. Features and objects shown in zone 1 are included in the formal category, while others shown in the rest part of the image, from zone 2 to zone 6 , are included in the latter category.

A detailed list of anomalies, their main features, and criteria for labeling in the image are shown in table 1 . We built and trained a deep learning model to detect signs of those 17 diseases from an image. It covers a wide region of panoramic radiography and will reduce the burden of clinicians in hospitals and help the prevention and early diagnosis of diseases.

Table 1. Category, name, and labeling criteria of 17 fine-grained dental anomalies

\begin{tabular}{|c|c|c|}
\hline Category & Name of anomaly & Description \\
\hline \multirow{4}{*}{$\begin{array}{l}\text { Soft tissue } \\
\text { calcification }\end{array}$} & Calcified carotid atherosclerotic plaque & Irregular linear radiopacity. \\
\hline & Lymph node calcification & Irregular or cauliflower-like radiopacity, One or multiple \\
\hline & Ossification of the stylohyoid ligament & Long and thin radiopacity in anteroinferior direction \\
\hline & Tonsillar calcification & $\begin{array}{l}\text { One or multiple radiopacities in the dorsal surface of the } \\
\text { tongue }\end{array}$ \\
\hline \multirow[t]{4}{*}{$\begin{array}{l}\text { Carious } \\
\text { lesions }\end{array}$} & Cervical caries or abrasion & $\begin{array}{l}\text { Notch or half-moon shaped radiolucency in the cervical area } \\
\text { of the tooth }\end{array}$ \\
\hline & Dental caries or coronal defect & $\begin{array}{l}\text { Various patterns of radiolucency in occlusal surface of the } \\
\text { tooth }\end{array}$ \\
\hline & Proximal caries & $\begin{array}{l}\text { Various patterns of radiolucency in the interproximal surface } \\
\text { of the tooth }\end{array}$ \\
\hline & Secondary caries & $\begin{array}{l}\text { Various patterns of radiolucency in the inferior area of } \\
\text { restorations }\end{array}$ \\
\hline \multirow{4}{*}{$\begin{array}{l}\text { Anomalies } \\
\text { in the dental } \\
\text { region }\end{array}$} & External root resorption & Irregular shape of the root \\
\hline & Impacted tooth & $\begin{array}{l}\text { A condition in which the tooth is not normally erupted and is } \\
\text { ambushed in the bone }\end{array}$ \\
\hline & Periapical radiolucency & $\begin{array}{l}\text { Various patterns of radiolucency in the periapical area of the } \\
\text { tooth }\end{array}$ \\
\hline & Residual root & Loss of coronal portion in tooth \\
\hline
\end{tabular}




\begin{tabular}{|l|l|l|}
\hline \multirow{2}{*}{} & Supernumerary tooth & $\begin{array}{l}\text { Tooth in addition to the normal series of deciduous or } \\
\text { permanent dentition }\end{array}$ \\
\cline { 2 - 3 } & Tooth overlapped with mandibular canal & $\begin{array}{l}\text { The root of the third molar is overlapped with the mandibular } \\
\text { canal }\end{array}$ \\
\hline $\begin{array}{l}\text { Anomalies } \\
\text { in } \\
\text { surrounding } \\
\text { region }\end{array}$ & Mucosal thickening in maxillary sinus & Various patterns of radiopaque shadow in maxillary sinus \\
\cline { 2 - 3 } & Radiopacity in jaw & Various patterns of radiopacity in jaw \\
\cline { 2 - 3 } & Retention pseudocyst in maxillary sinus & Dome-shaped radiopaque shadow in maxillary sinus \\
\hline
\end{tabular}

\section{Result}

\section{Dataset generation}

A large amount of high-quality datasets is one of the essential factors to take advantage of machine learning techniques. To build a model that is directly applicable to real clinical practices, we installed a data transfer module in imaging devices in local dental clinics, and automatically collected panoramic dental images from the device to our cloud server directly. In this study, a total of 22,999 panoramic dental images were collected from 30 local dental clinics during a year, from July 2020 to July 2021. All image data were fully anonymized and deidentified and the study is performed in accordance with the Declaration of Helsinki.

The size of image datasets used in previous studies varies from 87 to 3,000 according to the review [14]. Compared with those datasets, the size of our dataset exceeds the size of the others. The dataset is manually labeled by a dental radiography expert. The data labeling process is highly laborious and time-consuming. We parallelly labeled images during the collection period. Figure 1 shows some examples of labeled images. 

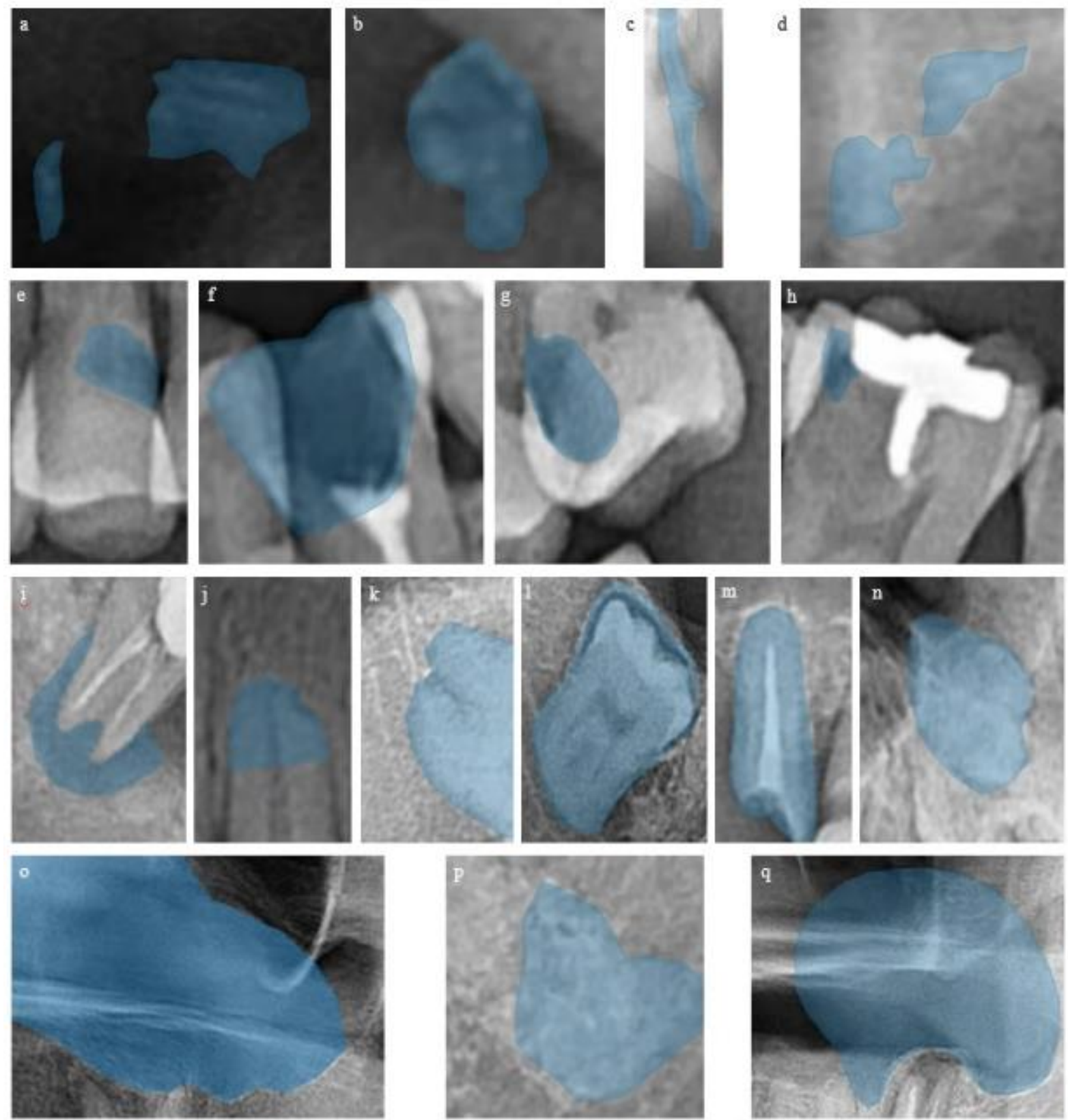

Figure 1. Examples of labeled 17 dental anomalies. a) Calcified carotid atherosclerotic plaque, b) Lymph node calcification, c) Ossification of stylohyoid ligament, d) Tonsillar calcification, e) Cervical caries or abrasion, f) Dental caries or coronal defect, g) Proximal caries, h) Secondary caries, i) Periapical radiolucency, j) External root resorption, k) Tooth overlapped with mandibular canal, l) Impacted tooth, m) Residual root, n) Supernumerary tooth, o) Mucosal thickening on maxillary sinus, p) Radiopacity in jaw, and q) Retention pseudocyst on maxillary sinus. 


\section{Anomaly detection}

Our detection model consists of four parts (Fig 2.a). First, we convert DICOM formatted images directly transferred from dental clinics into PNG format. Then it detects anomalies through trained faster R-CNN models [19]. Basically it is an object detection model, we got object boxed regions of high possibilities of anomalies (Fig 2.b). Next we filter out some boxes that are not located in a predetermined region (Fig 2.c). For example, if detected boxes are about carious lesions, it is obvious that the boxes should be located in the dental region. However, if some of the boxes are not located in the region but in the surrounding region, we can be sure that those boxes are absurd, so we can filter out those boxes. In the final stage, we narrow down the region of abnormal signs from a box form to a polygon (Fig 2.d). Through this stage dental clinicians can get high-resolution information not only the location and the type of anomalies found in the image, also the specific regions that show the feature of the anomalies. We used a prebuilt library, Detectron2 [20], in the polygon shaper stage. Figure 2.e is an example of our detection model. There are two signs of dental anomalies, proximal caries and periapical radiolucency. The proximal caries in the figure is in asymptotic stage so it may be stay undetected without careful examination. This model successfully detected it which means that the model can help early detection of selected anomalies.

We tested our detection model using part of our dataset. We built and trained our model using data taken from July 2020 to March 2021, and tested our model with images taken from April 2021 to July 2021. Table 2 shows the class-wise performance of our detection model applied to the test dataset. Our model shows very high specificity, in most classes over $95 \%$, which means it can filter out most of the normal or healthy images. High specificity means that this model can be used to reduce the burden of examination of dental clinicians because it successfully filters out healthy images so that clinicians can focus on other images. Precision and sensitivity vary depending on the type of anomalies but generally the score exceeds or is similar to that from previous studies.
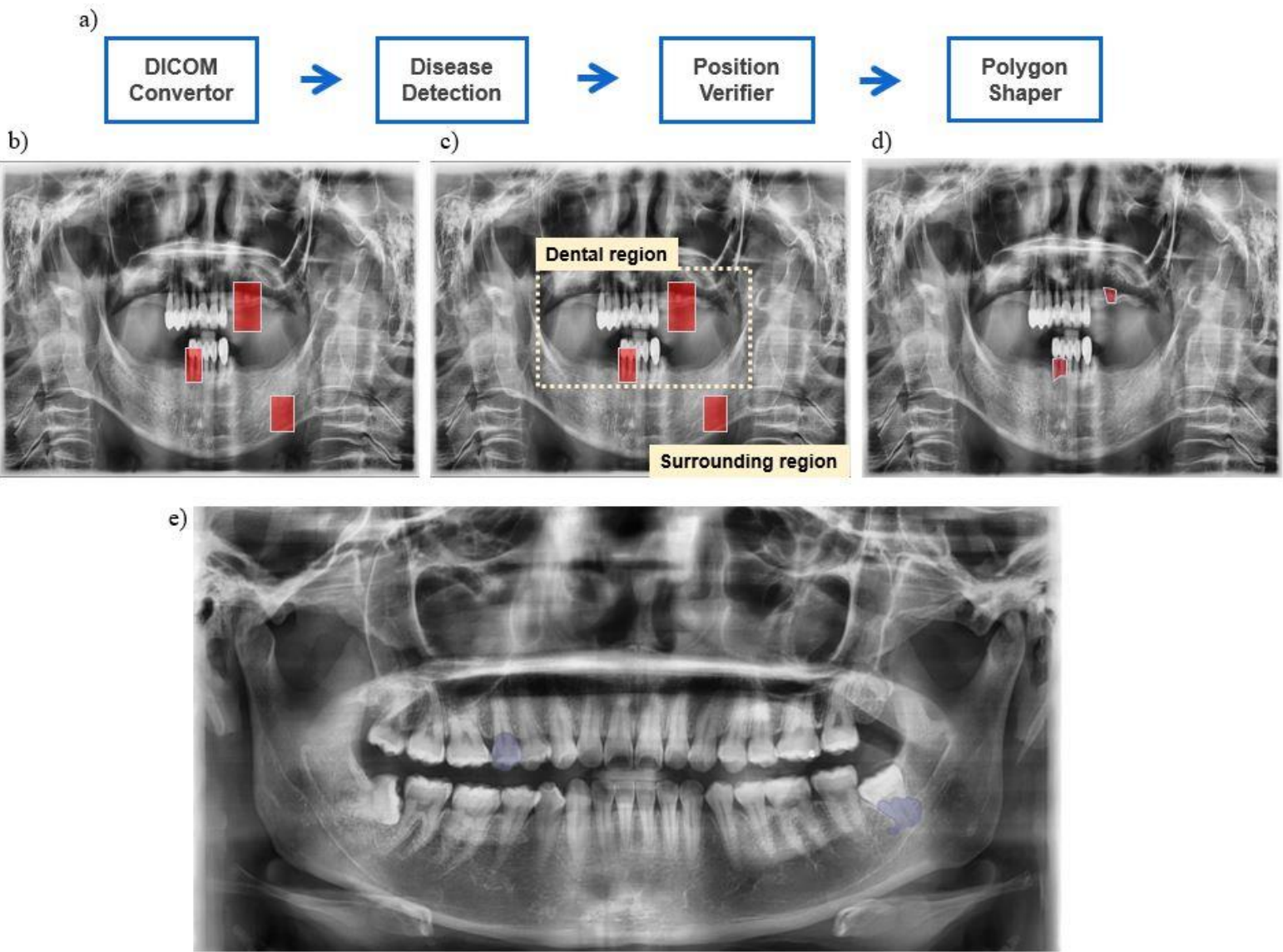
Figure 2. Anomaly detection process of our model. a) Overall process of this model, b) Result of disease detection stage. Boxes are found from the faster R-CNN architecture. c) Example of position verifier, carious lesion, for example, is known to be located in dental region, so boxes which are located in surrounding region are deleted. d) Result of polygon shaper. To notice dental clinicians why the regions are detected to be abnormal, this model narrows down the region into polygon shape. e) Example figure of final outcome of the model. Proximal caries in upper tooth is in asymptomatic stage but this model can find this. This model can contribute the early detection and diagnosis of several dental anomalies before they go severe, improving outcomes dental healthcare and patients' quality of life. 
Table 2. Class-wise performance score of the trained model

\begin{tabular}{|c|c|c|c|c|}
\hline Category & Name of anomaly & PRECISION & SENSITIVITY & SPECIFICITY \\
\hline \multirow[t]{4}{*}{$\begin{array}{l}\text { Soft tissue } \\
\text { calcification }\end{array}$} & Calcified carotid atherosclerotic plaque & 48.03922 & 95.71429 & 95.91049 \\
\hline & Lymph node calcification & 42.85714 & 27.27273 & 99.7006 \\
\hline & Ossification of the stylohyoid ligament & 72.59259 & 95.33333 & 97.02811 \\
\hline & Tonsillar calcification & 56.98925 & 100 & 96.90881 \\
\hline \multirow[t]{4}{*}{$\begin{array}{l}\text { Carious } \\
\text { lesions }\end{array}$} & Cervical caries or abrasion & 78.96825 & 76.51376 & 95.0421 \\
\hline & Dental caries or coronal defect & 78.43137 & 69.59064 & 98.20701 \\
\hline & Proximal caries & 47.9798 & 80.61224 & 91.55738 \\
\hline & Secondary caries & 47.61905 & 30.43478 & 99.15902 \\
\hline \multirow{6}{*}{$\begin{array}{l}\text { Anomalies } \\
\text { in the dental } \\
\text { region }\end{array}$} & External root resorption & 35.71429 & 33.33333 & 99.32836 \\
\hline & Impacted tooth & 68.38235 & 98.52941 & 92.56698 \\
\hline & Periapical radiolucency & 82.14971 & 95.08197 & 89.53881 \\
\hline & Residual root & 62.36559 & 89.32039 & 97.26135 \\
\hline & Supernumerary tooth & 31.37255 & 62.96296 & 97.35049 \\
\hline & Tooth overlapped with mandibular canal & 78.36257 & 100 & 93.1418 \\
\hline \multirow{3}{*}{$\begin{array}{l}\text { Anomalies } \\
\text { in } \\
\text { surrounding } \\
\text { region }\end{array}$} & Mucosal thickening in maxillary sinus & 57.46606 & 95.89041 & 92.257 \\
\hline & Radiopacity in jaw & 83.5443 & 97.94521 & 97.85479 \\
\hline & Retention pseudocyst in maxillary sinus & 62.85714 & 92.30769 & 99.01738 \\
\hline
\end{tabular}

\section{Discussion}

As we mentioned above, panoramic dental radiography is one of the most widely and frequently used imaging techniques in dentistry. It is safer than other imaging tools and quickly takes a wide range of dental structures so it is essential to make diagnoses and further treatment plans for patients, but due to the complicated structure of the dental region and lack of time, clinicians mainly focused on small parts of the images. If that neglected information is fully detected and noted to clinicians, it will improve the overall quality and consequences of the treatment. To achieve that goal, we applied an artificial intelligence technique which is widely used in the image analysis field to automatically detect regions of anomalies. We trained our model focused to increase the specificity to help clinicians filter out healthy panoramic dental radiography so as to decrease the number of images to be examined and to alleviate the burden of clinicians. First, we selected 17 major dental anomalies which are closely related to patients' oral health. These anomalies can lead to serious outcomes if ignored or are related to other systemic diseases. It means that the early detection of those anomalies can prevent severe outcomes and can be used as a marker to suspect other systemic diseases. We categorized anomalies into four groups corresponding to their clinical features and locations.

First, carious lesions are considered the most prevalent problem in dentistry [14]. Because carious lesions cause more serious problems if ignored, prevention and early diagnosis are very important. 
Many previous pieces of research applied artificial intelligence techniques to detect carious lesions. [13] Depending on its progress and location, various treatments can be used to treat carious lesions.

Here we divided carious lesions into four categories corresponding to their clinical features for fine-grained diagnoses; dental caries, cervical caries, proximal caries, and secondary caries. These subtypes of carious lesions show unique features which are related to their diagnoses and treatment. First, cervical caries is considered the most dangerous type because it leads to the rapid loss of tooth due to its location. Proximal caries is a type of carious lesion which is located on the surfaces between adjacent teeth. They are the most difficult type to detect because they cannot be visually or manually detected. Finally, secondary caries is a disease that occurs on the tooth after the filling. Because it takes a lot of burdens to detect. [21] This fine-grained diagnosis of carious lesions is important to early detection of caries before their progression to severe stages and to prevent further loss of dental tissues.

The second category of our fine-grained model, calcifications, occurs when calcium accumulates in body tissue. The diagnostic criteria of calcifications are their anatomical locations, distributions, numbers, sizes, and shapes. [22] Calcifications in maxillofacial areas can be found through examinations of panoramic dental radiography but there are very few studies conducted regarding them. [23] Though the presence of calcifications on panoramic dental radiographs is uncommon, their detection is important to prevent the further progression of diseases. We selected four calcification anomalies; Calcified carotid atherosclerosis plaque, lymph node calcifications, calcifications of the stylohyoid ligament, tonsillar calcifications (tonsilloliths) [24].

Our third category is dental anomalies. We included dental disease features and abnormal structures shown in the dental region [18] to this category. Dental anomalies are abnormal forms or structures of teeth in the dental area. We selected six dental anomalies which are critical factors of dental health; external root absorption, impacted tooth, periapical radiolucency, residual root, supernumerary tooth. Some of these anomalies often cause symptoms such as pain, halitosis, and bleeding, and can be used as diagnostic markers, and anatomical factors when planning further dental surgeries. For example, Periapical radiolucency is the radiographic changes around the apex of the tooth and is the sign of inflammatory bone lesions. Recent studies present that periapical radiolucency may be caused by several diseases such as cirrhosis [25]. External root resorption is an undesirable dental injury that causes a loss of some parts of a tooth and can be seen radiographically. This type of anomaly damages the underlying tissues and causes a number of complications including infection, loss of teeth, pain, and so on [26]. The positional relationship between the mandibular canal and corresponding tooth is a key anatomic factor to make surgical plans such as extraction of the mandibular third molar because damage to the inferior alveolar nerve affects the function of the stomatognathic system and the quality of life of patients [27]. Panoramic dental radiography is one way to evaluate the risk of nerve injury before the extraction [28]. Impacted teeth can cause several symptoms such as swollen gums, halitosis, and pain when opening the mouth. If ignored, it causes severe complications such as infection, cysts, absorption, and many gum diseases. A recent study presented that an impacted tooth might have some association with a large central osteoma [29]. A residual root is a leftover of a tooth in the jaw after an extraction. It sometimes causes infections and pain. Usually, it is recommended to extract with a local anesthetic. Finally, supernumerary teeth may lead to many severe problems like displacement, crowding, root resorption, dilaceration, loss of vitality of adjacent teeth, and even ameloblastomas and odontomas in severe cases. So clinicians should aware of the existence of the occurrence so that they can formulate treatment plans [30].

The last category is anomalies located in surrounding regions of the dental area. These anomalies are rarely related to oral health but may be used as potential markers to diagnose other related diseases. These radiographic anomalies are signs of inflammatory processes of that region and are known to be related to several diseases. For example, previous studies showed that retention pseudocysts of the maxillary sinus may have some associations with allergic and inflammatory processes, trauma, periapical and periodontal infections [31], radiopacity in jaws with many osteoblastic and osteoclastic activities [32], mucosal thickening of the maxillary sinus with apical periodontitis, alveolar bone loss, and so on [33].

To train a deep learning model to detect these many types of anomalies, it is essential to accumulate datasets including enough number of objects for every class. In fact, the most important factor of using artificial intelligence techniques is the quality and quantity of data. We built the system to collect panoramic dental images directly from local dental clinics and manually labeled them by a dental radiography expert. For a year, we accumulated a large and high-quality dataset compared to previous studies. This dataset is also still growing, so it 
has great potential in this field. Our tool successfully detects given anomalies with high performance especially for specificity and demonstrates that artificial intelligence can reduce the burden of dental clinicians by reducing the number of images that should be examined manually through detecting potential anomalies and filtering normal images.

\section{Method}

\section{Data acquisition}

Images are taken from 30 local dental clinics by panoramic $\mathrm{x}$-ray cameras model PaX-i, Rayscan alpha from VATECH, Papaya 3d from Genoray, and RealScan from PointNix. Images are fully anonymized removing all patient identifiers. We installed a data transfer module in imaging devices to automatically collect panoramic dental images from clinics. Images are collected from July 2020 to July 2021. For each image, above 17 anomalies are manually labeled one by one by a dental radiology expert. Image regions showing features of anomalies are first labeled in box form and then in the polygonal form to specify the exact region of the anomalies During the data collection period, data collection and labeling anomalies are done in parallel.

\section{Model architecture and training}

Because images are originally transferred in DICOM format, we converted images to PNG format. We used faster R-CNN architecture for dental anomaly detection [19]. We trained a model for every 17 dental anomalies. We divided our dataset into a train/validation set and a test set. All images taken from July 2020 to March 2021 are used as a train/validation set, and others which are taken from April 2021 to July 2021 are used as a test set. We built a position verifier to filter out wrongly detected object boxes from the R-CNN model. It takes each object class and its location and examines whether it is well detected or not. Two classes, Carious lesions and anomalies in the dental region, are expected to be in the dental region and other classes are expected to be in the surrounding region. Finally, in the polygon shaper stage, we used Detectron2 [20] to narrow down the detected regions from box form to polygon shape.

\section{Competing Interests}

D Kim and H Jeong are co-founders of an Invisionlab on dental medical analysis. The experiment, analysis, and interpretation of this study and its findings were not related to this. S Lee has no potential conflict of interest.

\section{Reference}

1. Rotemberg, V., Kurtansky, N., Betz-Stablein, B. et al. A patient-centric dataset of images and metadata for identifying melanomas using clinical context. Sci Data 8, 34 (2021). https://doi.org/10.1038/s41597-021-00815$\mathrm{z}$

2. Saad M Khan, Xiaoxuan Liu, Siddharth Nath, Edward Korot, Livia Faes, Siegfried K Wagner, et al. A global review of publicly available datasets for ophthalmological imaging: barriers to access, usability, and generalisability. The Lancet Digital Health, 2021, https://doi.org/10.1016/S2589-7500(20)30240-5

3. Amit Kumar Jaiswal, Prayag Tiwari, et al. Identifying pneumonia in chest X-rays: A deep learning approach. Measurement, vol 145, page 511-518, 2019, https://doi.org/10.1016/j.measurement.2019.05.076

4. D. Cai, X. Sun, N. Zhou, X. Han and J. Yao, Efficient Mitosis Detection in Breast Cancer Histology Images by RCNN. 2019 IEEE 16th International Symposium on Biomedical Imaging (ISBI 2019), 2019, pp. 919-922, doi: 10.1109/ISBI.2019.8759461.

5.Yüksel, A.E., Gültekin, S., Simsar, E. et al. Dental enumeration and multiple treatment detection on panoramic X-rays using deep learning. Sci Rep 11, 12342 (2021). https://doi.org/10.1038/s41598-021-90386-1 
6. Watanabe PCA, Faria V, Camargo AJ. Multiple Radiographic Analysis (Systemic Disease): Dental Panoramic Radiography. J Oral Health Dent Care 2017; 1:007

7. Kavitha, M S et al. Diagnosis of osteoporosis from dental panoramic radiographs using the support vector machine method in a computer-aided system. BMC medical imaging vol. 12 1. 16 Jan. 2012, doi:10.1186/14712342-12-1

8. Faure J, Engelbrecht A. Impacted tooth detection in panoramic radiographs. Advances in Computational Intelligence. IWANN 2021. Lecture Notes in Computer Science, vol 12861. Springer, Cham. https://doi.org/10.1007/978-3-030-85030-2_43 (1.1)

9. Oliveira, J.P.R. Caries detection in panoramic dental x-ray images. Master thesis, Universidade da Beira Interior Departamento de Informatica. 2009

10. Divya K Veena, Anand Jatti, Revan Joshi, Krishna S Deepu. Characterization of dental pathologies using digital panoramic x-ray images based on texture analysis. Annual International Conference of the IEEE Engineering in Medicine and Biology Society. vol. 2017 (2017): 592-595. doi:10.1109/EMBC.2017.8036894

11. Rad A.E., Amin I.B.M., Rahim M.S.M., Kolivand H. Computer-Aided Dental Caries Detection System from X-Ray Images. In: Phon-Amnuaisuk S., Au T. (eds) Computational Intelligence in Information Systems. Advances in Intelligent Systems and Computing, vol 331. Springer, Cham. 2015. https://doi.org/10.1007/978-3-319-131535_23

12. Vinayahalingam, S., Kempers, S., Limon, L. et al. Classification of caries in third molars on panoramic radiographs using deep learning. Sci Rep 11, 12609 (2021). https://doi.org/10.1038/s41598-021-92121-2

13. Arman, H., Mahdiyar, M. M., Seok-Bum, K., PaXNet: Dental caries detection in panoramic x-ray using ensemble transfer learning and capsule classifier, arXiv, cs.CV, 2020.

14. Prados-Privado, María et al. "Dental Caries Diagnosis and Detection Using Neural Networks: A Systematic Review.” Journal of clinical medicine vol. 9,11 3579. 6 Nov. 2020, doi:10.3390/jcm9113579

15. Kim, J., Lee, HS., Song, IS., et al. DeNTNet: Deep Neural Transfer Network for the detection of periodontal bone loss using panoramic dental radiographs. Sci Rep 9, 17615 (2019). https://doi.org/10.1038/s41598-019$53758-2$

16. Yang, Hyunwoo et al. Deep Learning for Automated Detection of Cyst and Tumors of the Jaw in Panoramic Radiographs. Journal of clinical medicine vol. 9,6 1839. 12 Jun. 2020, doi:10.3390/jcm9061839

17. Lee, Jae-Seo et al. Osteoporosis detection in panoramic radiographs using a deep convolutional neural network-based computer-assisted diagnosis system: a preliminary study. Dento maxillo facial radiology vol. 48,1 (2019): 20170344. doi:10.1259/dmfr.20170344

18. Langland OE, Langlais RP, Preece JW. Principles of dental imaging. 2nd edition. Baltimore, MD. Lippincott Williams \& Wilkins. 2002

19. Shaoqing Ren, Kaiming He, Ross Girshick, and Jian Sun. 2015. Faster R-CNN: towards real-time object detection with region proposal networks. In Proceedings of the 28th International Conference on Neural Information Processing Systems - Volume 1 (NIPS'15). MIT Press, Cambridge, MA, USA, 91-99.

20. Yuxin $\mathrm{Wu}$ and Alexander Kirillov and Francisco Massa and Wan-Yen Lo and Ross Girshick. Detectron2.https://github.com/facebookresearch/detectron2. 2019.

21. Brouwer F, Askar H, Paris S, Schwendicke F. Detecting Secondary Caries Lesions: A Systematic Review and Meta-analysis. Journal of Dental Research. 2016;95(2):143-151. doi:10.1177/0022034515611041

22. White S, Pharoah M. Radiología Oral: principios e interpretación. 1st ed. Barcelona: Harcourt; 2002. pp. 55265

23. Ivonne G, Henrique D N, Sergio O. Soft tissue calcified in mandibular angle area observed by means of panoramic radiography. Int J Clin Exp Med. 2014;7(1): 51-56. 
24. Babu B B, Tejasvi M L A, Avinash CK, B C. Tonsillolith: a panoramic radiograph presentation. J Clin Diagn Res. 2013;7(10):2378-2379. doi:10.7860/JCDR/2013/5613.3530

25. Lea et al. Presence and consequence of tooth periapical radiolucency in patients with cirrhosis. Hepat Med. 2016; 8 97-103

26. Patel Bobby. Root Resorption. Endodontic treatment, retreatment, and surgery: mastering clinical practice. 389-413. Springer International Publishing. Cham. https://doi.org/10.1007/978-3-319-19476-9_16

27. Gu L, Zhu C, Chen K, Liu X, Tang Z. Anatomic study of the position of the mandibular canal and corresponding mandibular third molar on cone-beam computed tomography images. Surg Radiol Anat. 2018;40(6):609-614. doi:10.1007/s00276-017-1928-6

28. Sedaghatfar M, August MA, Dodson TB. Panoramic radiographic findings as predictors of inferior alveolar nerve exposure following third molar extraction. J Oral Maxillofac Surg. 2005;63:3-7. doi: 10.1016/j.joms.2004.05.217.

29. Bhatt, G., Gupta, S., Ghosh, S. et al. Central Osteoma of Maxilla Associated with an Impacted Tooth: Report of a Rare Case with Literature Review. Head and Neck Pathol 13, 554-561 (2019). https://doi.org/10.1007/s12105-018-0994-3

30. Parolia A, Kundabala M, Dahal M, Mohan M, Thomas MS. Management of supernumerary teeth. J Conserv Dent. 2011;14(3):221-224. doi:10.4103/0972-0707.85791

31. Donizeth-Rodrigues C, Fonseca-Da Silveira M, Gonçalves-De Alencar AH, Garcia-Santos-Silva MA, Francisco-De-Mendonça E, Estrela C. Three-dimensional images contribute to the diagnosis of mucous retention cyst in maxillary sinus. Med Oral Patol Oral Cir Bucal. 2013;18(1):e151-e157. Published 2013 Jan 1. doi:10.4317/medoral.18141

32. Silva BSF, Bueno MR, Yamamoto-Silva FP, Gomez RS, Peters OA, Estrela C. Differential diagnosis and clinical management of periapical radiopaque/hyperdense jaw lesions. Braz Oral Res. 2017 Jul 3;31:e52. doi: 10.1590/1807-3107BOR-2017.vol31.0052. PMID: 28678971.

33. Song Ren, Haijiao Zhao, Jingbo Liu, Qingxuan Wang, Yaping Pan. Significance of maxillary sinus mucosal thickening in patients with periodontal disease. International Dental Journal. Vol 65, Issue 6, 303-310. 2015. https://doi.org/10.1111/idj.12186olith: A panoramic radiograph presentation. J Clin Diagn Res. 2013 Oct; 7(10): 2378-2379. 